\title{
Abnormal Ventral Frontal Response During Performance of an Affective Go/No Go Task in Patients with Mania
}

\author{
Rebecca Elliott, Alan Ogilvie, Judy S. Rubinsztein, Gloria Calderon, Raymond J. Dolan, and \\ Barbara J. Sahakian
}

\begin{abstract}
Background: Patients with mania show a behavioral bias toward positive information in an emotional go/no go task. This is the converse of the bias toward negative information seen in unipolar depression that we have recently related to the abnormal function of ventral and medial prefrontal cortices (PFCs). The aim of this study was to investigate the neuronal basis of the bias toward positive information in manic patients.
\end{abstract}

Methods: During performance of an emotional go/no go task using functional magnetic resonance imaging, 8 manic patients and 11 healthy controls were scanned. The task allowed comparison between neural response to happy, sad, and neutral words in the context of these words being either targets or distracters.

Results: Manic patients showed attenuated orbitofrontal response when all the semantic conditions were compared with a control condition; however, they showed an enhanced response of the left ventrolateral PFC to emotional relative to neutral targets, as well as enhanced ventral and medial prefrontal response to emotional, particularly happy, distracters.

Conclusions: These results suggest a critical role for ventral and medial dysfunction in the pathology of mania, which might underpin aspects of cognitive and clinical symptomatology.

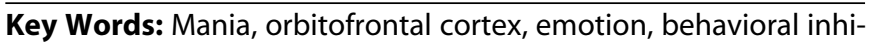
bition, fMRI

$\mathrm{I}$ $\mathrm{n}$ a recent study, we reported that abnormal neuronal responses in medial and ventral prefrontal cortices (PFCs) were associated with performance of an emotional go/no go task in patients with recurrent unipolar depression (Elliott et al 2002). We suggested that these regions may represent a neural substrate for mood-congruent processing biases that have been reported in various cognitive contexts in unipolar depression (Bradley et al 1996; Williams et al 1997). The observation that depressed patients selectively process sad information is wellestablished, however a recent neuropsychological study using an emotional go/no go paradigm additionally reports a contrasting bias toward happy information in manic patients (Murphy et al 1999). A similar bias has been demonstrated in emotion recognition tasks, where manic patients show impaired recognition of negative emotions (Lembke and Ketter 2002), in contrast to depressed patients, who tend to rate negative expressions as more intense than controls (Hale 1998); however, a bias toward happy information has not always been observed, with Lyon et al (1999) suggesting that manic patients show biases toward both positive and negative stimuli depending on social context. The aim of the present study was to determine whether an affective bias toward emotional information in manic patients is associated with abnormal ventral and medial prefrontal function.

There are relatively few functional imaging studies of manic patients, possibly because of the difficulties in imaging this

From the Neuroscience and Psychiatry Unit (RE), University of Manchester, Manchester, United Kingdom; Department of Psychiatry (AO), University of Oxford, Oxford, United Kingdom; Department of Psychiatry (JSR, GC, BJS), University of Cambridge, Cambridge, United Kingdom; and Wellcome Department of Cognitive Neurology (RJD), London, United Kingdom.

Address reprint requests to Dr. Rebecca Elliott, Neuroscience and Psychiatry Unit, Room G907, Stopford Building, University of Manchester, Oxford Road, Manchester, United Kingdom, M13 9PT.

Received October 8, 2003; revised February 27, 2004; accepted March 3, 2004. clinically unstable group of patients. An early study using single photon emission computed tomography (SPECT) with a relatively large group of medicated manic patients showed that there were no significant differences compared with controls (Silfverskiold and Risberg 1989). Goodwin et al (1997) reported increased orbitofrontal activation in a SPECT study of a group of patients becoming manic following lithium withdrawal. Using positron emission tomography (PET), Baxter and colleagues (Baxter et al 1985) did not show significant changes in whole brain metabolism in unmedicated manic subjects compared with controls; however, they did show that when bipolar depressed or mixed-state patients were rescanned in the euthymic or hypomanic state, there was significantly higher whole brain metabolism in the more euphoric state compared with the depressed state (Baxter et al 1985). By contrast, other studies have found evidence of hypofrontality in manic compared with control subjects (Al Mousawi et al 1996; Rubin et al 1995; Soares and Mann 1997; Stoll et al 2000). There have also been observations of increased subcortical metabolism in the basal ganglia, thalamus (Baxter et al 1989), and amygdala (Al Mousawi et al 1996). Drevets et al (1997) used $\mathrm{H}_{2}{ }^{15} \mathrm{O}$ PET to measure regional cerebral blood flow abnormalities associated with mania and reported focal increases in activation of subgenual cingulate cortex. The same group also reported reduced structural volume in this region in mania (Drevets et al 1998) and a reduction of subgenual glial cells at postmortem. A relationship between cingulate activation and manic state has been suggested by Blumberg et al (1999), who reported enhanced cingulate activations in patients during the manic stage relative to the euthymic stage of illness.

There have been very few attempts to use functional imaging in conjunction with cognitive activation paradigms in patients with mania. Blumberg et al (2000) have described reduced activation of rostral and orbitofrontal cortices (OFCs) in response to a word generation task, as well as reduced activation at rest. Rubinsztein et al (2001) reported a mixed pattern of prefrontal response associated with a decision-making task, with enhanced dorsal cingulate response, but attenuated response in frontopolar cortex and inferior frontal gyrus. Using more emotional stimuli, Yurgelun-Todd et al (2000) observed abnormal responses to 
facial expressions in a mixed group of stable outpatients with bipolar disorder. Patients showed normal responses to happy faces, but reduced dorsolateral prefrontal and enhanced amygdala activation to fearful faces.

Thus, the limited functional imaging literature using cognitive activation paradigms in mania is consistent with the hypothesis that prefrontal regions may be functionally abnormal in response to a cognitive challenge, and the results of Yurgelun-Todd suggest that affective valence may be a factor in differential prefrontal function. In normal subjects, the processing of emotional material (emotional faces, affective pictures and film clips) has been associated with neuronal responses in ventral and medial regions of the PFC (Dolan et al 1996; Lane et al 1998; Lane et al 1999; Iwase et al 2002). In a study using an emotional version of the Stroop paradigm, Whalen et al (1998) reported that the ventral portion of the anterior cingulate was differentially responsive to emotionally toned words. In our previous study using the affective go/no go paradigm, both the ventral and subgenual cingulate were differentially activated by emotional words in control subjects (Elliott et al 2000).

In depressed patients we found that ventral regions of anterior cingulate responded selectively to sad rather than happy words, the opposite pattern to that seen in control subjects (Elliott et al 2002). This directly reflected the processing bias toward sad words reported by Murphy et al (1999). Since the behavioral performance of manic patients on the affective go/no go task shows exactly the opposite bias to depressed patients (Murphy et al 1999), it is logical to hypothesize that in manic patients the normal differential ventral cingulate response to happy words might be exaggerated. Our previous study also reported that depressed patients showed differential responses in lateral OFC to affectively toned (particularly sad) distracters, a finding not seen in controls. We hypothesize that the same effect may be present in manic patients but with a bias toward happy rather than sad distracters, in line with the behavioral findings of Murphy et al (1999).

\section{Methods and Materials}

\section{Subjects}

A total of 8 right-handed patients with a diagnosis of bipolar depressive disorder were recruited from inpatient and outpatients services in Cambridge, 4 male and 4 female. Diagnosis was established using a structured interview and case note review. Patients had to fulfil research diagnostic criteria and DSM-IV criteria for bipolar affective disorder and be manic at the time of screening. Patients with a history of neurologic disease or closed head injury were excluded, as were those with a concurrent diagnosis of other psychiatric disorder or substance abuse.

The mean age was 33.5 years and the mean score on the Young mania rating scale (Young et al 1978) was 28.1 (range 13-49). Of the 8 subjects, 7 were clinically considered to meet criteria for bipolar disorder with a current manic episode and 1 with a current hypomanic episode according to DSM-IV criteria established using the Schedule for Affective Disorder and Schizophrenia (Lifetime Version; SADS-L; Spitzer and Endicott; 1977). All but 1 subject who was hypomanic were on psychotropic medication: 5 were on lithium carbonate with levels maintained in the therapeutic range $\left(.6-1.0 \mathrm{mmol}^{-1}\right) ; 2$ were on sodium valproate $(1,200 \mathrm{mg} /$ day and 2,400 $\mathrm{mg} /$ day); 5 patients had concomitant antipsychotic therapy with their mood stabilizer: 2 were on chlorpromazine at $350 \mathrm{mg} / \mathrm{day}, 1$ at $450 \mathrm{mg} / \mathrm{day}, 1$ on risperidone at $6 \mathrm{mg} / \mathrm{day}$, and 1 on zuclopenthixol hydrochloride at $14 \mathrm{mg} / \mathrm{day} ; 2$ had concomitant benzodiazepines: clonazepam at $18 \mathrm{mg}$ /day and lorazepam at $2 \mathrm{mg}$ /day with a mood stabilizer; 1 had received concomitant sodium amytal at $180 \mathrm{mg} /$ day, and 1 patient was taking $7.5 \mathrm{mg}$ zopiclone at night. Mean age of onset of bipolar disorder was 24.25 years and, excluding 1 subject who had a rapid cycling disorder with too many depressive and manic episodes to count, the mean number of manic episodes was 2.57 and the mean number of depressive episodes was 1.57 . Out of 8 patients, 5 had a lifetime history of psychotic symptoms during either a manic or depressive episode and 2 were suffering from psychotic symptoms (grandiose delusions) at the time of the scan. Subjects had a mean Mini Mental State Examination (Folstein et al 1975) score of 29.75 (out of 30) and a mean National Adult Reading Test (NART) IQ of 111.8 (SD \pm 8.4 ).

These patients were compared with 11 right-handed volunteers who were recruited by advertisement in the local community ( 8 women and 3 men; age range $24-59$, mean age 37.6, SD \pm 9.7 years; mean NART IQ 113.4, SD \pm 6.3 ). The results obtained from this volunteer group have been reported in a previous study (Elliott et al 2000). The groups did not differ significantly in terms of age or IQ. Controls were screened using the Beck Depression Inventory and verbal interview to exclude anyone with current affective symptomatology or a history of neurologic or psychiatric disorder, head injury, or substance abuse.

The study was approved by local research ethics committees (Joint Ethics Committees of National Hospital and Institute of Neurology, London, and the Addenbrookes Hospital Research Ethics Committee, Cambridge). Informed written consent was obtained from all subjects before scanning.

\section{Cognitive Activation Paradigm}

The paradigm used is discussed in detail elsewhere (Elliott et al 2000; Elliott et al 2002) and was the same for both subject groups. This was a blocked design study with 24 blocks of the active task interspersed with 24 blocks of rest. The task in each block was based on a classic go/no go methodology where subjects have to respond as quickly as possible to target stimuli and withhold responses to distracter stimuli. In the version used here, all stimuli were words. In the main task conditions, targets and distracters were defined on the basis of emotional valence; with either happy, sad, or neutral words as targets, presented with one of the other categories as the distracters (e.g., happy targets with sad distracters). The words in each category were matched for word length and frequency (Hofland and Johansson 1982), and the neutral words were selected so that they were not readily imageable.

A lower level control condition was also included, where all the words were neutral and the targets and distracters were defined on the basis of physical rather than semantic properties (italic font vs. plain text). Overall we used 8 task conditions: 1) happy targets, sad distracters; 2) happy targets, neutral distracters; 3) sad targets, happy distracters; 4) sad targets, neutral distracters; 5) neutral targets, happy distracters; 6) neutral targets, sad distracters; 7) italic targets, plain distracters; 8) plain targets, italic distracters. Conditions 1-6 assessed the effects of attending to different emotional tone, allowing the effects of differential responses to the valence of both targets and distracters to be addressed. Conditions 7 and 8 provided a perceptuomotor control condition where all words were neutral and no semantic judgment was required.

In each block, 10 targets and 10 distracters were presented in a randomized order. Each word was shown for $300 \mathrm{msec}$, with a 
Table 1. The Mean Behavioral Scores on 3 Performance Measures: Reaction Times, Mean Error Rate (False Positives) per 20-Word Block and Mean Omissions (Nonresponse to Targets) per 20-Word Block

\begin{tabular}{|c|c|c|c|c|c|c|}
\hline & \multicolumn{3}{|c|}{ Controls } & \multicolumn{3}{|c|}{ Patients } \\
\hline Mean Reaction Time in ms & $535(8.8)$ & 549 (7.9) & $554(9.3)$ & $531(9.5)$ & $556(9.6)$ & $550(8.5)$ \\
\hline Mean Omissions /Block & $.63(.09)$ & $.67(.07)$ & $.59(.07)$ & $.75(.13)$ & $.69(.12)$ & $.66(.09)$ \\
\hline
\end{tabular}

Standard errors of the mean are given in parentheses.

$\mathrm{ms}$, millisecond

900-msec interstimulus interval to allow subjects to respond (or not) by means of a button press with the right index finger. Each 24-sec task block was followed by a 24-sec rest block, at the end of which written instructions for the next task block were presented. There were 3 blocks of each task condition.

\section{MRI Scanning}

Patients and controls were scanned by the same team of staff, on the same scanner, and with identical cognitive task and scanning parameters. Data were acquired on a $2 \mathrm{~T}$ Siemens VISION system (Siemens AG, London, United Kingdom). A gradient echo echo-planar $\mathrm{T}^{*}$ sequence was used to acquire functional images continuously with a repetition time ( $\mathrm{Tr}$ ) of 4 sec. 294 images were acquired for each subject, with each image comprising 48 axial slices at 3 -mm separation, allowing whole brain coverage. In plane resolution was $3 \mathrm{~mm} \times 3 \mathrm{~mm}$. The first 6 volumes of each run were dummy volumes to allow for T1-weighted equilibration effects and were discarded before analysis. A T1-weighted structural scan was also acquired for each subject, for coregistration purposes and to exclude any gross structural pathology. No such pathology was seen in any subject.

\section{Data Analysis}

For consistency, data were analyzed in the same way as for our previous studies (Elliott et al 2000; Elliott et al 2002) using Statistical Parametric Mapping (SPM99; Wellcome Department of Cognitive Neurology, London, United Kingdom). The SPM methodology has been discussed in considerable detail elsewhere (see www.fil.ion.ucl.ac.uk/spm for full reference list). Functional scans for each subject were first realigned using the first as a reference image. They were then normalized into a standard stereotactic space using MNI (Montreal Neurologic Institute) templates and the coordinate system of Talairach and Tournoux (1988), and spatially smoothed using a 10-mm Gaussian kernel to facilitate intersubject averaging. A random effects model was used to analyze the data statistically. For each subject, one mean image was generated for each of the 9 conditions ( 8 task conditions plus rest). These mean images were combined in a series of linear contrasts to assess group effects. Statistical parametric maps of the T statistic (SPM[T]) were generated for each comparison, and these were converted to a normal distribution (SPM[Z]). In line with established functional imaging conventions, we report neuronal responses observed at an uncorrected statistical threshold of $p<.001$ for regions about which we had prior anatomical hypotheses. For descriptive purposes we also report results significant at this threshold in regions for which there was no prior hypothesis; however, interpretation and discussion is limited to hypothesized areas (medial and ventral frontal cortices), and those where neuronal responses were observed at $p<.05$ corrected for multiple comparisons. Anatomical designations are based on the atlas of Duvernoy (1991) and the structural magnetic resonance images of the groups.

\section{Results}

Performance data are given in Table 1 . There were no significant differences in terms of accuracy or reaction times.

For clarity, the imaging results reported in detail below are the results of between-group comparisons (bipolar patients vs. controls). The aim of this study was to identify regions where bipolar patients showed significantly enhanced or attenuated neuronal responses relative to controls. Including within-group analysis does not directly address this question. The within-group analysis in controls has been published previously (Elliott et al 2000), and the details of the within-group analysis in manic patients is available from the authors on request.

\section{All Go/No Go Conditions Compared with Rest}

This comparison represents conditions 1-8 combined and compared with the rest condition (Table 2). Patients with mania showed attenuated neuronal response in the left middle frontal gyrus (BA 9). Patients showed enhanced response in bilateral superior temporal gyrus (BA 22), but inspection of adjusted blood oxygenation level-dependent (BOLD) response data suggested that this was due to elevated responses in these regions in control subjects during rest blocks rather than a genuine elevation in patients during the active task.

Table 2. Regions Showing Between-Group Differences For Go/No Go Performance Compared with Rest

\begin{tabular}{|c|c|c|c|c|c|c|c|}
\hline \multicolumn{8}{|l|}{ Controls-Patients } \\
\hline $\begin{array}{l}\text { Middle Frontal gyrus } \\
\text { Patients-Controls }\end{array}$ & L & 9 & -45 & 6 & 36 & 4.03 & 164 \\
\hline \multirow[t]{2}{*}{ Superior temporal gyrus } & L & 22 & -60 & -12 & 6 & 3.78 & 101 \\
\hline & $\mathrm{R}$ & 22 & 63 & -6 & 3 & 3.19 & 249 \\
\hline
\end{tabular}

$Z$ values are thresholded at 3.09, corresponding to a significance level of $p<.001$, uncorrected. Coordinates are for the maximally activated voxel in each cluster. Cluster sizes are the number of contiguously activated voxels. Sample sizes were $n=11$ for controls and $n=8$ for patients. 
Table 3. Regions Showing Between-Group Differences for Semantic Versus Orthographic Go/No Task

\begin{tabular}{|c|c|c|c|c|c|c|c|}
\hline Region & Left/Right & Brodmann's Area & \multicolumn{3}{|c|}{ Talairach Coordinates } & $z$ & Cluster Size \\
\hline \multicolumn{8}{|l|}{ Controls-Patients $^{a}$} \\
\hline Inferior temporal gyrus & L & 20 & -54 & -12 & -21 & 4.13 & 268 \\
\hline Inferior occipital gyrus & $\mathrm{R}$ & 18 & 33 & -93 & -6 & 3.51 & 118 \\
\hline \multirow[t]{2}{*}{ Inferior frontal gyrus ${ }^{a}$} & $\mathrm{~L}$ & 47 & -54 & 33 & -6 & 3.39 & 43 \\
\hline & $\mathrm{R}$ & 47 & 51 & 30 & -12 & 3.09 & 27 \\
\hline \multirow[t]{2}{*}{ Middle frontal gyrus } & $\mathrm{R}$ & 10 & 51 & 48 & 6 & 4.17 & 83 \\
\hline & $\mathrm{R}$ & 10 & 24 & 60 & 3 & 3.65 & 225 \\
\hline
\end{tabular}

$Z$ values are thresholded at 3.09, corresponding to a significance level of $p<.001$, uncorrected. Coordinates are for the maximally activated voxel in each cluster. Cluster sizes are the number of contiguously activated voxels. Sample sizes were $n=11$ for controls and $n=8$ for patients.

${ }^{a}$ Region for which there was a prior hypothesis and is therefore subsequently discussed.

\section{Semantic Compared with Orthographic Conditions}

This comparison represents conditions 1-6 compared with 7 and 8 (Table 3). Compared with normal control subjects, manic patients showed attenuated neuronal responses in bilateral inferior temporal gyrus (BA 20), right inferior occipital gyrus (BA 18), and bilateral inferior frontal gyrus (BA 47) extending from ventrolateral PFC to lateral OFC (Figure 1). In all these regions there was a lower response to the orthographic conditions in the control subjects. Put another way, the response to the semantic targets was similar for the two groups, but the differential response relative to the orthographic targets was reduced in the patient group.

Enhanced neuronal response in patients relative to controls was observed in precuneus (BA 7) and regions of right frontopolar cortex (BA 10). Adjusted BOLD response data suggest that frontopolar regions showed greater response in the orthographic relative to semantic conditions in control subjects and the reverse pattern in patients (a crossover interaction between condition

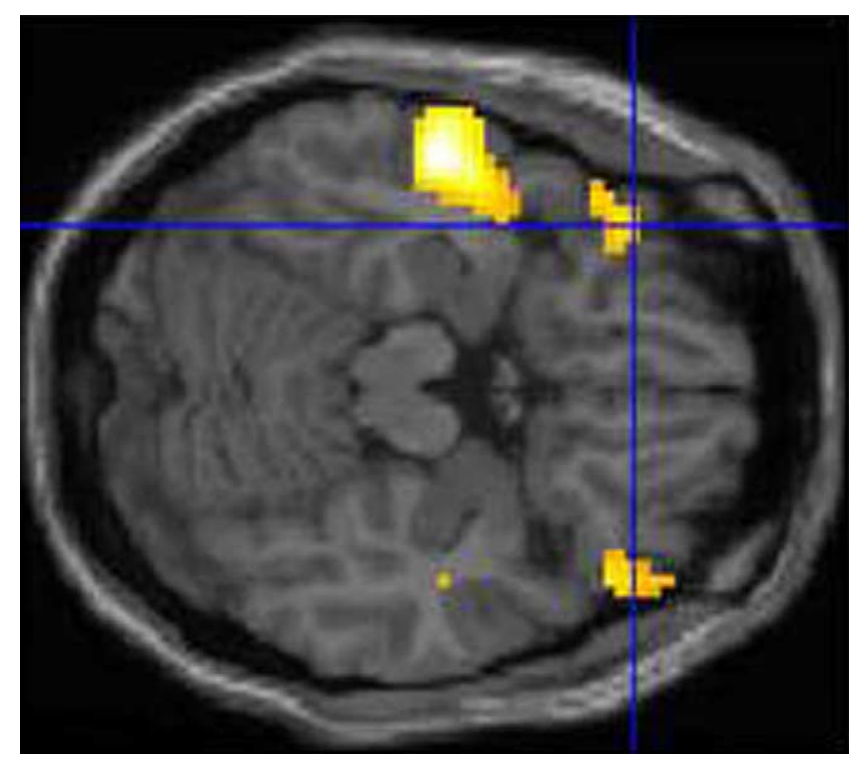

Figure 1. Enhanced bilateral orbitofrontal response in controls relative to patients for the semantic compared with the orthographic task. This suggests hypofunction of this region in go/no go performance in manic patients. Blood oxygenation level-dependent response thresholded at $p<$ .001 unconnected (corresponding to $Z=309$ ) and superimposed onto a template structural magnetic resonance image. and diagnosis). By contrast, the precuneus was significantly less responsive in the control condition for the patient group.

\section{Emotional Compared with Neutral Targets}

This comparison represents conditions 1-4 compared with 5 and 6 (Table 4). Manic patients compared with controls showed attenuated neuronal responses associated with emotional targets relative to neutral targets in right cuneus (BA 19) and right middle frontal gyrus (BA 46). In these regions, adjusted BOLD response data suggested that the differential effect was in fact due to enhanced response to the neutral targets in manic patients.

In the reverse contrast, manic patients showed elevated neuronal responses associated with emotional relative to neutral targets in right inferior occipital gyrus (BA 19), left inferior frontal gyrus (BA 47), left inferior temporal gyrus (BA 20), right middle frontal gyrus (BA 9), and a superior frontal polar region (BA 8). The frontal responses were all due to a crossover interaction between valence and diagnosis, such that controls showed enhanced response in the neutral conditions while patients showed enhanced response in the emotional conditions.

When happy and sad targets were compared separately with neutral targets, enhanced response in left inferior frontal gyrus (BA 47) and right middle frontal gyrus was observed for both, but only at a subthreshold level of significance. Thus, this effect is present for both happy and sad targets, but only when they are combined in the "emotional versus neutral" subtraction does the effect reach statistical significance.

It should be noted that the emotional versus neutral comparison described above is not matched for distracter valence. However, when we compared conditions 1 and 3 with conditions 5 and 6 such that distracter valence was matched, the same pattern of results was observed.

\section{Happy Versus Sad Targets}

This comparison represents conditions 1 and 2 compared with 3 and 4 . There were no differences between patients and controls that reached significance at $p<.001$ uncorrected.

\section{Differential Effects of Distracters}

Control subjects showed no differential effects of distracter types on neuronal response; however, manic patients showed enhanced response to sad relative to neutral distracters in right dorsolateral (BA 9/46) and ventrolateral (BA 44/45) PFCs. They also showed enhanced responses to happy distracters in bilateral dorsolateral PFC (BA 46) and subgenual cingulate extending to medial OFC (BA 10; Table 5 and Figure 2). The same pattern of 
Table 4. Regions Showing Between-Group Differences for Emotional Compared with Neutral Targets

\begin{tabular}{|c|c|c|c|c|c|c|c|}
\hline Region & Left/Right & Brodmann's Area & \multicolumn{3}{|c|}{ Talairach Coordinates } & $z$ & Cluster Size \\
\hline \multicolumn{8}{|l|}{ Controls-Patients $^{a}$} \\
\hline Cuneus & $\mathrm{R}$ & 19 & 12 & -90 & 36 & 4.29 & 183 \\
\hline \multicolumn{8}{|l|}{ Patients-Controls ${ }^{a}$} \\
\hline Inferior occipital gyrus & $\mathrm{R}$ & 19 & 45 & -81 & -9 & 3.99 & 180 \\
\hline Inferior frontal gyrus ${ }^{a}$ & $\mathrm{~L}$ & 11 & -42 & 42 & -12 & 3.69 & 29 \\
\hline Superior frontal gyrus & $\mathrm{L}$ & 8 & -24 & 51 & 42 & 3.29 & 71 \\
\hline
\end{tabular}

$Z$ values are thresholded at 3.09, corresponding to a significance level of $p<.001$, uncorrected. Coordinates are for the maximally activated voxel in each cluster. Cluster sizes are the number of contiguously activated voxels. Sample sizes were $n=11$ for controls and $n=8$ for patients.

${ }^{a}$ Region for which there was a prior hypothesis and is therefore subsequently discussed.

responses was also observed when individual conditions matched for target valence were contrasted (e.g., condition 1 vs. condition 2 or condition 3 vs. condition 4$)$

\section{Effects of Demographic and Clinical Variables}

Since the groups were not matched for gender balance, the effects of gender on the above results were explicitly modeled. There were no significant effects of gender in any of the regions discussed. We also performed a correlational analysis in the manic patients, using their score on the Young Mania Rating Scale as a covariate. None of the activations reported showed a significant correlation with symptom severity. Given the range of medication regimes, it was not possible to perform any systematic investigation of the effects of medication.

\section{Discussion}

The results of this study suggest that manic patients with bipolar affective disorder show abnormal patterns of neuronal response associated with performance of an affective go/no go task. When all go/no go conditions were compared with rest, there were only subtle differences between the groups; however, the contrasts of most interest are those comparing different emotional conditions, where global attentional demands are matched. Manic patients showed attenuated ventrolateral frontal response when all the semantic conditions were compared with the orthographic controls; however, they showed an enhanced response of the left ventrolateral region to emotional relative to neutral targets, as well as enhanced prefrontal responses to emotional distracters.

The contrast of all go/no go conditions compared with rest was associated with attenuated activation in the left medial frontal gyrus in patients. The patients showed no performance deficits on the task, and therefore this attenuated activation is not associated with significant attentional deficits as measured by this particular test of focused attention. The patients were not assessed neuropsychologically as part of this study, and it is possible that a more stringent test of focused attention would have revealed behavioral impairments. Hypoactivity in the middle frontal gyrus may play a role in attentional function in manic patients; however, this is beyond the scope of the present study. The key comparisons here were between the different emotional conditions of the same task; thus we were looking at whether affective valence differentially modulated attentional performance, using contrasts that control for any absolute difference in global attentional response.

Patients with mania demonstrated an attenuated neural response in ventrolateral PFC, extending to lateral OFC, when semantic targets were compared with orthographic targets. This effect was due to neuronal response to the orthographic task in patients being enhanced relative to controls, such that there was less difference between the orthographic and semantic conditions. Performance of go/no go tasks places demands on behavioral inhibition processes, in that prepotent responses to irrelevant distracters must be suppressed. Functional imaging studies have suggested that ventral frontal regions, including lateral OFCs, are critically involved in go/no go performance (Garavan et al 2002.; Kawashima et al 1996; Casey et al 1997; Horn et al 2003), and activity of these regions may depend on how difficult it is to inhibit responding. In the present study, controls showed greater ventrolateral PFC response to semantic than orthographic

Table 5. Regions Showing Between-Group Differences for Sad and Happy Distractors

\begin{tabular}{|c|c|c|c|c|c|c|c|}
\hline Region & Left/Right & Brodmann's Area & \multicolumn{3}{|c|}{ Talairach Coordinates } & $Z$ & Cluster Size \\
\hline \multicolumn{8}{|l|}{ Patients-Controls ${ }^{a}$} \\
\hline \multicolumn{8}{|l|}{ Sad distractors ${ }^{a}$} \\
\hline Inferior frontal gyrus & $\mathrm{R}$ & 47 & 60 & 12 & 0 & 3.80 & 143 \\
\hline \multicolumn{8}{|l|}{ Patients-Controls ${ }^{a}$} \\
\hline \multicolumn{8}{|l|}{ Happy distractors ${ }^{a}$} \\
\hline Subgenual cingulate gyrus ${ }^{a}$ & $\mathrm{~L}$ & 24 & -3 & 30 & -6 & 3.51 & 54 \\
\hline Medial frontal gyrus ${ }^{a}$ & $L / R$ & 10 & 0 & 42 & -9 & 3.17 & 104 \\
\hline
\end{tabular}

$Z$ values are thresholded at 3.09, corresponding to a significance level of $p<.001$, uncorrected. Coordinates are for the maximally activated voxel in each cluster. Cluster sizes are the number of contiguously activated voxels. Sample sizes were $n=11$ for controls and $n=8$ for patients.

${ }^{a}$ Region for which there was a prior hypothesis and is therefore subsequently discussed. 

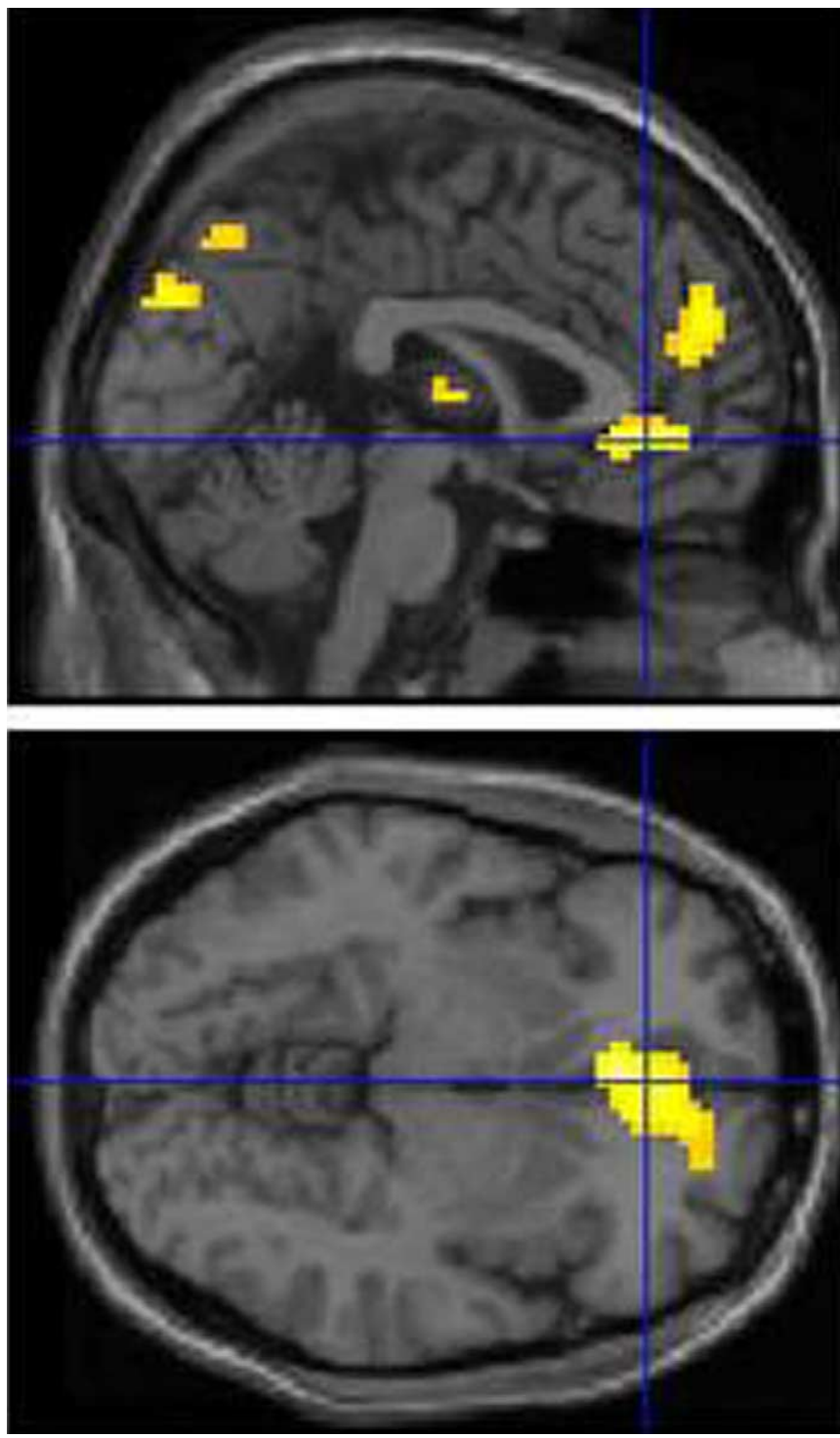

Figure 2. Enhanced medial orbitofrontal response in patients relative to controls for happy relative to neutral distracters. This suggests hyperfunction of this region in manic patients in response to mood-congruent taskirrelevant information. Blood oxygenation level-dependent response threshold at $p<.001$ uncorrected (corresponding to $Z=309$ ) and superimposed onto a template structural magnetic resonance image.

targets, suggesting that they were working harder to inhibit irrelevant information under the semantic condition. For the manic patients, the easier orthographic conditions elicited ventrolateral PFC response comparable to the level of response seen for the semantic condition. It may be that patients have to work harder to suppress irrelevant information in the orthographic condition, perhaps suggesting that they are processing semantic information even though it is unnecessary to do so.

Differential ventrolateral PFC response, specifically on the left, was seen in patients when emotional and neutral targets were compared within the semantic domain. This was found to reflect enhanced response to emotional relative to neutral targets in patients, and the effect was reversed in controls. The effect generalized to both happy and sad emotions with no valencespecificity observed, and was also observed when distracter valence was matched. There were no differences in neuronal response when happy and sad targets were directly compared. The subtle difference in neuronal response when affective valence of semantic targets was varied suggests that the functioning of putative behavioral inhibition processes could depend on emotional properties of stimuli. Manic patients showed relatively enhanced ventral frontal function when targets were emotional, but attenuated function when targets were neutral. Our a priori hypothesis, based on the neuropsychological study of Murphy et al (1999) was that patients might show a bias toward positive targets; in fact, our results suggest a bias toward both positive and negative targets relative to neutral. This would be consistent with the finding of Lyon et al (1999), who reported biases toward both positive and negative stimuli in manic patients.

The most pronounced differences between manic patients and controls were observed when responses to different distracter valences were compared. Control subjects show no differential responses to distracter valence. In our earlier study of unipolar depressives, we reported that emotional distracters, particularly sad distracters, elicited differential lateral OFC responses when target valences were matched. For patients with mania, sad relative to neutral distracters were associated with differential response in right dorso- and ventrolateral frontal regions, but not OFC regions. Happy relative to neutral distracters were also associated with right lateral frontal regions. Additionally, however, response was observed in a region extending from subgenual cingulate cortex to medial OFC. When happy and sad distracters were compared directly, the conditions were distinguished only by differential subgenual/medial OFC response to happy distracters.

It therefore appears that irrelevant aspects of stimuli capture neuronal resources in manic patients. Both happy and sad distracters were associated with differential responses in both dorso- and ventrolateral PFCs. This suggests that emotional distracters are attended to a greater extent than neutral distracters and may place greater demands on inhibition processes mediated by ventrolateral prefrontal regions. The contrast between happy and sad distracters provides the only evidence for mood congruent effects in this study; with happy distracters eliciting additional response in subgenual cingulate and medial OFC. In the detailed neuropsychological study by Murphy et al (1999), a version of the emotional go/no go task was used with no neutral stimuli. Happy words were paired with sad distracters and vice versa. The reported presence of mood-congruent bias in manic patients actually manifested itself as significantly slower response to sad targets, a pattern that is consistent with the hypothesis that patients find it more difficult to inhibit responses to moodcongruent (happy) distracters, since sad targets were always paired with happy distracters. The present results support this hypothesis to a certain extent; however, there are more similarities than differences between responses to happy and sad stimuli, implicating a more general effect of emotional valence. It has been argued (Clark et al 2001; Harmer et al 2002) that distractibility and impairment of sustained attention may represent core neuropsychological deficits associated with mania. The results presented here, in conjunction with the neuropsychological results of Murphy et al (1999), suggest an affective dimension to this impairment. Distracting information with emotional relevance may capture neuronal resources, increasing subjects' inability to focus on the task in hand.

These findings in patients with mania show interesting contrasts with our previous findings in patients with unipolar depression (Elliott et al 2002). Depressed patients showed no 
significant differences in neuronal response when semantic targets were compared with orthographic targets. By contrast, the manic patients showed enhanced ventrolateral PFC response in the orthographic control conditions. This may reflect a greater generalized distractibility in manic patients, such that inhibition processes need to be engaged at an easier level of the task due to distracting effects of irrelevant semantic information. When the affective valence of targets was compared, depressed patients showed attenuated neuronal responses to emotional targets (happy and sad) in subgenual cingulate and a posterior region of lateral OFC. By contrast, manic patients showed enhanced response in a more dorsal and anterior region of the ventrolateral PFC. This would be consistent with blunted emotional response in depressed patients and exaggerated emotional response in manic patients, compatible with the clinical profile of these groups; however, it is important to note that these effects are mediated by distinct functional regions in the two populations, suggesting distinct mechanisms. Further, depressed patients showed differential response of the rostral cingulate for sad relative to neutral target words, consistent with the processing bias reported by Murphy et al (1999). Counter to our a priori hypothesis, manic patients did not show the opposite effect. The lack of differential response to happy and sad target valence in patients with mania is compatible with a nonconservative bias toward emotional stimuli in general.

Both manic and unipolar depressed patient groups showed differential lateral frontal responses to both happy and distracter stimuli relative to controls. This may suggest that problems inhibiting responses to task-irrelevant emotional information is common to depression and mania; however, in depressed patients the response was substantially ventral to that observed in patients with mania, again suggesting distinct functional mechanisms. There was also some evidence for mood congruency in responses to distracters. When happy and sad distracters were compared directly, depressed patients showed enhanced right lateral OFC response to sad distracters but no differential enhanced response to happy distracters. For patients with mania, the comparison revealed enhanced response to happy distracters in subgenual cingulate extending to medial OFC but no differential enhanced response to happy distracters. This might account for the behavioral effects described by Murphy et al (1999), but it is clearly simplistic to describe mania and depression as characterized by opposite biases to distracters, given the very different functional regions mediating these effects. While both groups do show differential responses dependent on affective valence of distracters as well as targets, these patterns are complex and distinct.

There are a number of limitations of the present study. The sample group is relatively small, due to the practical difficulties of scanning patients with mania; there may be results that do not reach significance here that would do so in a larger group. It is also possible that significant correlations with clinical features may be observed in a larger group. Patients were not assessed using a neuropsychological battery, and therefore it is not possible to relate the effects observed here to generalized deficits in attention or other aspects of cognitive function. Also, we did not include a measure of residual depressive symptomatology, so we cannot assess whether that some residual, and possibly transient, depressive symptoms may have contributed to the effects we observed. Finally, as is often the case with imaging studies of psychiatric populations, the patients were taking a range of different medications. Given the range of medication regimes involved in the small group of patients, it was not possible to perform any systematic analysis of medication effects.

Despite these caveats, the study still provides clear evidence that affective valence significantly modulates cognitive processing in patients with mania. Differential responses in PFC, particularly ventral and medial regions, mediate this affective modulation. The pattern observed in manic patients contrasts with our previous findings in patients with unipolar depression, in that both happy and sad information appears to distract patients from the task in hand at a neuronal level. It remains to be seen whether this is a trait effect in bipolar affective disorder or a state-dependent effect specific to the manic phase. Studies examining responses in manic, euthymic, and depressed patients with bipolar disorder would be needed to address this question.

The research was supported by a Wellcome Trust Program Grant No. 019407 to T.W. Robbins, B.J. Everitt, A.C. Roberts, and BJS. We are grateful to Dr. Paul Fletcher for help with scanning and for useful comments on a draft manuscript.

Al Mousawi AH, Evans N, Ebmeier KP, Roeda D, Chaloner F, Ashcroft GW (1996): Limbic dysfunction in schizophrenia and mania. A study using 18F-labelled fluorodeoxyglucose and positron emission tomography. BrJPsychiatry 169:509-516.

Baxter LR Jr, Phelps ME, Mazziotta JC, Schwartz JM, Gerner RH, Selin CE, Sumida RM (1985): Cerebral metabolic rates for glucose in mood disorders. Studies with positron emission tomography and fluorodeoxyglucose F 18. Arch Gen Psychiatry 42:441-447.

Baxter LR Jr, Schwartz JM, Phelps ME, Mazziotta JC, Guze BH, Selin SE (1989): Reduction of prefrontal cortex glucose metabolism common to three types of depression. Arch Gen Psychiatry 46:243-250.

Blumberg HP, Stern E, Martinez D, Ricketts S, de Asis J, White T, et al (2000): Increased anterior cingulate and caudate activity in bipolar mania. Biol Psychiatry 48:1045-52.

Blumberg HP, Stern E, Ricketts S, Martinez D, de Asis J, White T, et al (1999): Rostral and orbital prefrontal cortex dysfunction in the manic state of bipolar disorder. Am J Psychiatry 156:1986-1988.

Bradley BP, Mogg K, Millar N (1996): Implicit memory bias in clinical and non-clinical depression. Behav Res Ther 34:865-879.

Casey BJ, Trainor RJ, Orendi JL, Schubert AB, Nystrom LE, Giedd JN, et al (1997): A developmental functional MRI study of prefrontal activation during performance of a go-no-go task. J Cogn Neurosci 9:835-847.

Clark L, Iversen SD, Goodwin GM (2001): A neuropsychological investigation of prefrontal cortex involvement in acute mania. Am J Psychiatry 158:1605-11.

Dolan RJ, Fletcher P, Morris J, Kapur N, Deakin JF, Frith CD (1996): Neural activation during covert processing of positive emotional facial expressions. Neuroimage 3:194-200.

Drevets WC, Ongur D, Price JL (1998): Neuroimaging abnormalities in the subgenual prefrontal cortex: Implications for the pathophysiology of familial mood disorders. Mol Psychiatry 3:220-226.

Drevets WC, Price JL, Simpson JR Jr, Todd RD, Reich T, Vannier M, Raichle ME (1997): Subgenual prefrontal cortex abnormalities in mood disorders. Nature 386:824-827.

Duvernoy HM (1991): The Human Brain: Surface, Three-Dimensional Sectional Anatomy and MRI. New York: Springer-Verlag.

Elliott R, Rubinsztein JS, Sahakian BJ, Dolan RJ (2000): Selective attention to emotional stimuli in a verbal go/no go task: An fMRI study. Neuroreport 11:1739-1744.

Elliott R, Rubinsztein JS, Sahakian BJ, Dolan RJ (2002): The neural basis of mood-congruent processing biases in depression. Arch Gen Psychiatry 59:597-604.

Folstein MF, Folstein SE, MacHugh PR (1975): Mini Mental State Examination. Lutz, Florida: Psychological Assessment Resources A.

Garavan H, Ross TJ, Murphy K, Roche RAP, Stein EA (2002): Dissociable executive functions in the dynamic control of behaviour: Inhibition, error detection and correction. Neuroimage 17:1820-1829.

Goodwin GM, Cavanagh JT, Glabus MF, Kehoe RF, O'Carroll RE, Ebmeier KP (1997): Uptake of $99 \mathrm{mTc}$-exametazime shown by single photon emis- 
sion computed tomography before and after lithium withdrawal in bipolar patients: Associations with mania. Br J Psychiatry 170:426-430.

Hale WW 3rd (1998): Judgment of facial expressions and depression persistence. Psychiatry Res 80:265-274.

Harmer CJ, Clark L, Grayson L, Goodwin GM (2002): Sustained attention deficit in bipolar disorder is not a working memory impairment in disguise. Neuropsychologia 40:1586-90.

Hofland K, Johansson S (1982): Word Frequencies in British and American English. Bergen: The Norwegian Computing Centre for the Humanities.

Horn NR, Dolan M, Elliott R, Deakin JFW, Woodruff PWR (2003): Response inhibition and impulsivity: An fMRI study. Neuropsychologia 41:19591966.

Iwase M, Ouchi Y, Okada H, Yokoyama C, Nobezawa S, Yoshikawa E, et al (2002): Neural substrates of human facial expression of pleasant emotion induced by comic films: A PET Study. Neuroimage 17:758-768.

Kawashima R, Satoh K, Itoh H, Ono S, Furumoto S, Gotoh R, et al (1996): Functional anatomy of GO/NO-GO discrimination and response selection-a PET study in man. Brain Res 728:79-89.

Lane RD, Chua PM, Dolan RJ (1999): Common effects of emotional valence, arousal and attention on neural activation during visual processing of pictures. Neuropsychologia 9:989-997.

Lane RD, Reiman EM, Axelrod B, Yun LS, Holmes A, Schwartz GE (1998): Neural correlates of levels of emotional awareness. Evidence of an interaction between emotion and attention in the anterior cingulate cortex. $J$ Cognitive Neuroscience 10:525-535.

Lembke A, Ketter TA (2002): Impaired recognition of facial affect in mania. Am J Psychiatry 159:302-304.

Lyon HM, Startup M, Bentall RP (1999): Social cognition and the manic defense: Attributions, selective attention and self-schema in bipolar affective disorder. J Abnorm Psychol 108:273-282.

Murphy FC, Sahakian BJ, Rubinsztein JS, Michael A, Rogers RD, Robbins TW,
Paykel ES (1999): Emotional bias and inhibitory control processes in mania and depression. Psychol Med 29:1307-1321.

Rubin E, Sackeim HA, Prohovnik I, Moeller JR, Schnur DB, Mukherjee S (1995): Regional cerebral blood flow in mood disorders: IV. Comparison of mania and depression. Psychiatry Res 61:1-10.

Rubinsztein JS, Fletcher PC, Rogers RD, Ho LW, Aigbirhio FI, Paykel ES, et al (2001): Decision-making in mania: A PET study. Brain 124:25502563.

Silfverskiold P, Risberg J (1989): Regional cerebral blood flow in depression and mania. Arch Gen Psychiatry 46:253-259.

Soares JC, Mann JJ (1997): The anatomy of mood disorders-review of structural neuroimaging studies. Biol Psychiatry 41:86-106.

Spitzer RL, Endicott J (1977): A diagnostic interview: The schedule for affective disorders and schizophrenia. Arch Gen Psych 35:837-844.

Stoll AL, Renshaw PF, Yurgulen-Todd DA, Cohen BM (2000): Neuroimaging in Bipolar Disorder: What have we learned? Biol Psychiatry 48:505517.

Talairach J, Tournoux P (1988): Co-planar stereotactic atlas of the human brain. New York: Thieme.

Whalen PJ, Bush G, McNally RJ, Wilhelm S, McInerney SC, Jenike MA, Rauch SL (1998): The emotional counting Stroop paradigm: A functional magnetic resonance imaging probe of the anterior cingulate affective division. Biol Psychiatry 44:1219-1228.

Williams JMG, Watts FM, Macleod C, Mathews A (1997): Cognitive Psychology and Emotional Disorders, 2nd ed. New York: John Wiley and Sons Ltd.

Young RC, Biggs JT, Ziegler VE, Meyer DA (1978): A Rating Scale for Mania: Reliability, Validity and Sensitivity. Br J Psychiatry 133:429-435.

Yurgelun-Todd DA, Gruber SA, Kanayama G, Killgore WD, Baird AA, Young $A D$ (2000): $f M R I$ during affect discrimination in bipolar affective disorder. Bipolar Disorders 2:237-248. 